\title{
Scheme-independent calculations of anomalous dimensions of baryon operators in conformal field theories
}

\author{
John A. Gracey, ${ }^{1}$ Thomas A. Ryttov, ${ }^{2}$ and Robert Shrock ${ }^{3}$ \\ ${ }^{1}$ Theoretical Physics Division, Department of Mathematical Sciences, University of Liverpool, \\ P.O. Box 147, Liverpool L69 3BX, United Kingdom \\ ${ }^{2} \mathrm{CP}^{3}$-Origins, University of Southern Denmark, Campusvej 55, DK-5230 Odense M, Denmark \\ ${ }^{3}$ C. N. Yang Institute for Theoretical Physics and Department of Physics and Astronomy, \\ Stony Brook University, Stony Brook, New York 11794, USA
}

(Received 7 May 2018; published 22 June 2018)

\begin{abstract}
We present the first analytic scheme-independent series calculations of anomalous dimensions of several types of baryon operators at an infrared fixed point (IRFP) in an asymptotically free SU(3) gauge theory with $N_{f}$ fermions. Separately, for an asymptotically free gauge theory with a gauge group $G$ and $N_{f}$ fermions in a representation $R$ of $G$, we consider physical quantities at an IRFP, including the anomalous dimension of gauge-invariant fermion bilinears and the derivative of the beta function. These quantities have been calculated in series expansions whose coefficients have been proved to be scheme-independent at each order. We illustrate the scheme independence using a variety of schemes, including the RI' scheme and several types of momentum subtraction schemes.
\end{abstract}

DOI: 10.1103/PhysRevD.97.116018

\section{INTRODUCTION}

In conformal field theories, quantities of particular interest are the scaling dimensions, $D_{\mathcal{O}}$, of gauge-invariant operators, $\mathcal{O}$. In general, we write

$$
D_{\mathcal{O}}=D_{\mathcal{O}, c l .}-\gamma_{\mathcal{O}}
$$

where $D_{\mathcal{O}, c l .}$ is the classical (free-field) dimension of $\mathcal{O}$, and $\gamma_{\mathcal{O}}$ is the anomalous dimension of $\mathcal{O}$ due to interactions. We shall focus on the determination of $\gamma_{\mathcal{O}}$ in perturbation theory at a fixed point of the renormalization group (RG). An important example of such a fixed point is encountered in the case of an asymptotically free nonAbelian gauge theory with gauge group $G$ and sufficiently many massless fermions in a representation $R$ of $G$. We denote the running gauge coupling at a Euclidean scale $\mu$ as $g=g(\mu)$ and denote $\alpha=g^{2} /(4 \pi)$ and $a=g^{2} /\left(16 \pi^{2}\right)$. In this theory, the gauge coupling evolves from small values in the ultraviolet (UV) at large $\mu$ to an infrared fixed point (IRFP) at a value denoted $\alpha_{\mathrm{IR}}$ as $\mu \rightarrow 0$. At this value, the theory is scale-invariant and is inferred to be conformally invariant [1,2]. This infrared behavior is commonly denoted the non-Abelian Coulomb phase (NACP) or

Published by the American Physical Society under the terms of the Creative Commons Attribution 4.0 International license. Further distribution of this work must maintain attribution to the author(s) and the published article's title, journal citation, and DOI. Funded by SCOAP ${ }^{3}$. conformal window. The RG evolution of the gauge coupling is described by the beta function,

$$
\beta_{\alpha}=\frac{d \alpha}{d \ln \mu}=-2 \alpha \sum_{\ell=1}^{\infty} b_{\ell} a^{\ell}
$$

where $b_{\ell}$ is the $\ell$-loop coefficient. At the two-loop $(2 \ell)$ level [3-7], the IR zero of $\beta_{\alpha}$ function occurs at

$$
\alpha_{\mathrm{IR}, 2 \ell}=-\frac{4 \pi b_{1}}{b_{2}}
$$

If $N_{f}$ is only slightly smaller than the upper limit,

$$
N_{u}=\frac{11 C_{A}}{4 T_{f}}
$$

implied by the property of asymptotic freedom $[5,6]$, then $\alpha_{\mathrm{IR}, 2 \ell}$ is small and can be analyzed perturbatively $[4,7]$. As $N_{f}$ decreases, the value of the coupling at the infrared zero of the beta function increases, motivating calculation of this IRFP value of $\alpha$ to higher-loop order. This was carried out to the four-loop level for general gauge group $G$ and fermion representation $R$ in [8-10], using $b_{3}$ [11] and $b_{4}$ [12] computed in the modified minimal subtraction scheme [13] for regularization and renormalization, denoted $\overline{\mathrm{MS}}$. (The minimal subtraction scheme was originally presented in [14].) Subsequently, the IRFP was calculated to the 
five-loop level [15], using $b_{5}$ in the $\overline{\mathrm{MS}}$ scheme [16,17]. Effects of scheme dependence were studied in [18-25].

The anomalous dimension of a gauge-invariant operator $\mathcal{O}$, evaluated at a zero of the beta function (hence an RG fixed point), is, in principle, measurable, and hence cannot depend on the scheme used for regularization and renormalization. However, this property is not maintained in a conventional finite-order perturbative calculation of the anomalous dimension of such an operator as a power series in the coupling $\alpha$,

$$
\gamma_{\mathcal{O}}=\sum_{\ell=1}^{\infty} c_{\mathcal{O}, \ell} a^{\ell}
$$

Once the perturbative expansion for $\gamma_{\mathcal{O}}$ is truncated at a finite order, scheme dependence is induced in the result for $\gamma_{\mathcal{O}}$. Only if one had the entire perturbative series available would the final result be guaranteed to be schemeindependent. Explicitly, to evaluate $\gamma_{\mathcal{O}}$ to finite order at an IRFP using Eqs. (1.2) and (1.5), one solves for the relevant zero of the $n$-loop beta function to obtain the $n$-loop value of $\alpha$ at this IRFP, denoted $\alpha_{\mathrm{IR}, n \ell}$ and then substitutes this into Eq. (1.5) to obtain the value at the IRFP, $\gamma_{\mathcal{O}, \text { IR }}$. However, beyond the lowest orders, the result is schemedependent, because of scheme dependence in both the higher-order $b_{\ell}$ and the $c_{\mathcal{O}, \ell}$ coefficients. The calculations of $\gamma_{\bar{\psi} \psi, \mathrm{IR}}$ to four-loop order in [8,9] and to five-loop order in [15] used the four-loop and five-loop coefficients $c_{\bar{\psi} \psi \mu, 4}$ [26] and $c_{\bar{\psi} \psi, 5}$ [27], respectively, calculated in the $\overline{\mathrm{MS}}$ scheme. This scheme dependence of higher-order perturbative calculations is, of course, not limited to these quantities, but is a generic property of higher-order calculations. For example, it is well known that higher-order calculations of differential and total cross sections in quantum chromodynamics (QCD) are also scheme-dependent.

Intuitively, one expects that as one increases the order of the perturbative computation, there is more schemeindependent information contained in $\gamma_{\mathcal{O}}$. This expectation is justified by the fact that higher-order QCD calculations used, e.g., to analyze data from the Fermilab Tevatron and CERN Large Hadron Collider showed less dependence on the scheme/scale than lower-order calculations [28]. Indeed, for many years there has been work on the construction and application of schemes in QCD designed to reduce the scheme and scale dependence in higher-order QCD calculations (e.g., [29-31]).

Ideally, one would use a method of perturbative calculation of physical quantities that manifestly preserves the scheme independence at each finite order in the series expansion. That is, one would like to extract the schemeindependent information that is contained in the schemedependent higher-order coefficients $b_{\ell}$ and $c_{\mathcal{O}, \ell}$. A key property of the IRFP in an asymptotically free gauge theory is that $\alpha_{\mathrm{IR}} \rightarrow 0$ as $N_{f}$ (considered to be generalized to real numbers [6]) approaches the upper limit, $N_{u}$, allowed by asymptotic freedom. It follows that one can reexpress a physical quantity such as $\gamma_{\mathcal{O}}$ at the IRFP as a series expansion in powers of the difference

$$
\Delta_{f}=N_{u}-N_{f},
$$

i.e.,

$$
\gamma_{\mathcal{O}}=\sum_{n=1}^{\infty} \kappa_{\mathcal{O}, n} \Delta_{f}^{n}
$$

Since $\Delta_{f}$ is obviously scheme-independent and so is $\gamma_{\mathcal{O}}$, each coefficient $\kappa_{\mathcal{O}, n}$ is also scheme-independent. Some early work based on this was in $[7,32]$.

Recently, extensive scheme-independent expansions for anomalous dimensions of a number of physical quantities have been calculated and analyzed in [33-42]. For asymptotically free vectorial gauge theories with gauge group $G$ and $N_{f}$ fermions transforming according to a representation $R$ of $G$, physical quantities of interest include the fermion bilinears $\bar{\psi} \psi$ and $\bar{\psi} \mathcal{T}{ }_{j} \psi$, where we suppress the sum over fermion flavor indices and $\mathcal{T}_{j}$ denotes a generator of the Lie algebra of $\mathrm{SU}\left(N_{f}\right)$. These have the same anomalous dimension [43]. We denote this anomalous dimension as $\gamma_{\bar{\psi} \mu \nu}$ and its evaluation at the IRFP as $\gamma_{\bar{\psi} \psi, \text { IR }}$. The schemeindependent series expansion of $\gamma_{\bar{\psi} \mu, \text { IR }}$ is written as

$$
\gamma_{\bar{\psi} \psi, \mathrm{IR}}=\sum_{n=1}^{\infty} \kappa_{n} \Delta_{f}^{n}
$$

In general, the calculation of the coefficient $\kappa_{n}$ in Eq. (1.8) requires, as inputs, the values of the $b_{\ell}$ for $1 \leq \ell \leq n+1$ and the $c_{\ell}$ for $1 \leq \ell \leq n$.

The derivative of the beta function evaluated at the IRFP,

$$
\beta_{\mathrm{IR}}^{\prime}=\left.\frac{d \beta_{\alpha}}{d \alpha}\right|_{\alpha=\alpha_{\mathrm{IR}}},
$$

is also a physical quantity and hence is scheme-independent [44]. Indeed, from the trace anomaly [45] $T_{\mu}^{\mu}=$ $\left[\beta_{\alpha} /(4 \alpha)\right] F_{\mu \nu}^{a} F^{a \mu \nu}$, where $F_{\mu \nu}^{a}$ is the field-strength tensor, it follows that the full scaling dimension of $F^{2} \equiv \operatorname{Tr}\left(F_{\mu \nu} F^{\mu \nu}\right)$, satisfies the relation [46]

$$
D_{F^{2}}=4+\frac{d \beta_{\alpha}}{d \alpha}-\frac{2}{\alpha} \beta_{\alpha},
$$

so that, at the IRFP, with $\beta_{\alpha}=0, \gamma_{F^{2} \text { IR }}=-\beta_{\mathrm{IR}}^{\prime}$, i.e., $\beta_{\mathrm{IR}}^{\prime}$ is equivalent to the anomalous dimension of $F^{2}$ evaluated at the IRFP. The scheme-independent series expansion of $\beta_{\mathrm{IR}}^{\prime}$ is written as

$$
\beta_{\mathrm{IR}}^{\prime}=\sum_{n=2}^{\infty} d_{n} \Delta_{f}^{n}
$$


(Note that $d_{1}=0$ for all $G$ and $R$.) In general, the calculation of the coefficient $d_{j}$ in Eq. (1.11) requires, as inputs, the values of the $b_{\ell}$ for $1 \leq \ell \leq j$. In addition to these calculations for vectorial gauge theories, Ref. [41] carried out schemeindependent calculations of $\beta_{\mathrm{IR}}^{\prime}$ for chiral gauge theories.

The results of the scheme-independent series expansions in [33-40] are useful for several reasons, which are also motivations for the present study. First, they give new information about fundamental properties of conformal field theories, namely anomalous dimensions at an IRFP in the non-Abelian Coulomb phase of an asymptotically free gauge theory. A second important use of these calculations pertains to the determination of the size of the NACP. The upper end of the NACP, as a function of $N_{f}$, is known and is equal to $N_{u}$. However, for nonsupersymmetric theories, the lower end, at a value that we denote as $N_{f, c r}$, is not known, and there is an intensive ongoing effort to determine $N_{f, c r}$ by means of lattice simulations [47,48]. Applying schemeindependent calculations of $\gamma_{\bar{\psi} \mu, \mathrm{IR}}$, Refs. [34-38,40] obtained estimates of $N_{f, c r}$ in a manner complementary to lattice gauge simulations. This was done using the monotonic increase of $\gamma_{\bar{\psi} \psi, \text { IR }}$ with decreasing $N_{f}$ that was shown by the scheme-independent calculations, in conjunction with the rigorous upper limit on $\gamma_{\bar{\psi} \psi, \mathrm{IR}}$ from conformal invariance, namely $\gamma_{\bar{\psi} \psi, \mathrm{IR}}<2$ [49]. A third application follows from the second, namely that a knowledge of $N_{f, c r}$ (for a given gauge group $G$ and fermion representation $R$ ) is necessary for the construction and study of quasiconformal theories of physics beyond the Standard Model (BSM), since these require $N_{f}$ to be slightly less than $N_{f, c r}$ in order to achieve the slow running of the gauge coupling and associated quasiconformal behavior. In turn, the dynamical breaking of the approximate dilatation invariance in these theories leads to a light approximate Nambu-Goldstone boson, the dilaton $[47,48,50-52]$. These vectorial BSM theories can naturally arise from the sequential breaking of asymptotically free chiral gauge theories [53]. This is relevant to the investigation of the Higgs boson; although its production and decay properties are consistent with the predictions of the Standard Model, there is the continuing question of whether it might be a composite, dilaton-like state resulting from a quasiconformal BSM theory [51].

The accuracy of the scheme-independent series expansions of $\gamma_{\bar{\psi} \psi, \mathrm{IR}}$ and $\beta_{\mathrm{IR}}^{\prime}$ was studied in several ways in [33-42]. One way was to evaluate the stability of these quantities as higher-order terms in powers of $\Delta_{f}$ were added in the series. It was shown that the finite-order scheme-independent series calculations were most accurate at the upper end of the NACP, and remained reasonably accurate over a substantial portion of the NACP extending to lower values of $N_{f}$.

For the gauge group $G=\mathrm{SU}(3)$, a baryon operator has the form of a product of three fermion fields, each transforming as the fundamental (triplet) representation of $G$, with their gauge indices $a, b, c$ contracted with the $\epsilon_{a b c}$ tensor to form a color singlet. Relevant previous studies of anomalous dimensions of baryon operators in QCD include [54-61]. In particular, the anomalous dimensions of baryon operators have been calculated to one-loop [54], two-loop $[55,57]$, and three-loop order [58,59] as powers series in $\alpha$ and related studies have been presented in $[60,61]$.

In this paper we shall present, for the first time, analytic scheme-independent series calculations to order $O\left(\Delta_{f}^{3}\right)$ of anomalous dimensions of several types of baryon operators at an infrared fixed point of an asymptotically free SU(3) gauge theory with $N_{f}$ fermions in the fundamental representation. An assessment of the accuracy of these calculations will also be given. As was discussed previously [33-35], the procedure for the calculation of schemeindependent series expansions requires that the IRFP be exact, and this is only the case in the non-Abelian Coulomb phase, in which the chiral flavor symmetry is exact [62]. Since we thus necessarily restrict our analysis to the NACP, where there is no confinement, we use the term "baryon" to refer only to the property that the baryon operators that we consider are singlets under the SU(3) gauge symmetry. We note that there is actually some irony in using the term "baryon" here, since it is derived from the Greek word

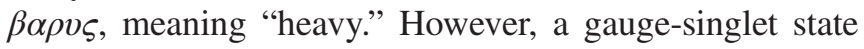
produced by the operation of a baryon creation operator on the vacuum in the non-Abelian Coulomb phase is massless, as are all physical states in this phase.

As a second part of our paper, we shall present, for general gauge group $G$ and fermion representation $R$, an explicit illustration of the scheme independence of the earlier calculations of $\Delta_{f}$ expansions of $\gamma_{\bar{\psi} \psi, \text { IR }}$ and $\beta_{\mathrm{IR}}^{\prime}[33-38,40]$. These calculations naturally used the $\overline{\mathrm{MS}}$ scheme because the $n$-loop coefficients in the beta function and in $\gamma_{\bar{\psi} \psi}$ had been calculated to the highest loop order in this scheme, and these coefficients have the simplest form in this scheme. Since a rigorous proof was already given in these earlier works of the scheme independence of the coefficients in these $\Delta_{f}$ expansions, it is not necessary to carry out the calculations in schemes other than the simplest one. However, it is, nevertheless, quite instructive to see how the considerably more complicated higher-order coefficients in the beta function and anomalous dimensions in these more complicated schemes combine to reproduce exactly the results of the $\overline{\mathrm{MS}}$ scheme for the coefficients in the various $\Delta_{f}$ series expansions. For the purpose of these illustrations, we shall consider a variety of different schemes, including the $\mathrm{RI}^{\prime}$ scheme $[63,64]$ and several varieties of momentum (MOM) subtraction schemes [29,65-70] (see also [71]).

It should be mentioned that this program of explicitly demonstrating scheme independence of the coefficients in the $\Delta_{f}$ expansions of anomalous dimensions of various operators was previously carried out for the $\mathcal{N}=1$ 
supersymmetric gauge theories in $[33,35,36,39]$, where it was shown that the use of two different schemes, namely the $\overline{\mathrm{DR}}$ scheme [72] and the Novikov-Shifman-VainshteinZakharov (NSVZ) scheme [73] yield the same schemeindependent results for the anomalous dimension of a holomorphic composite product of chiral superfields, $\gamma_{\Phi_{\text {comp }}, \mathrm{IR}}$, which, order by order are in precise agreement with the corresponding series expansion of the exactly known expression [74]. In addition to demonstrating explicitly that different schemes yield the same values of coefficients in the scheme-independent expansion of $\gamma_{\Phi_{\text {comp }} \text { IR }}$ of the form (1.7), this work showed that (i) the series (1.7) converges to the exact expression everywhere where the latter applies, i.e., in the NACP, (ii) for a fixed $N_{f}$ in the NACP, a finite truncation of the series (1.7) to order $O\left(\Delta_{f}^{p}\right)$ approaches the exact expression exponentially rapidly, and (iii) throughout the entire NACP, one achieves excellent accuracy of a few percent even with a series calculated to a modest order of $n=4$, i.e., $O\left(\Delta_{f}^{4}\right)$. These scheme-independent calculations of anomalous dimensions in an $\mathcal{N}=1$ supersymmetric gauge theory thus improved upon conventional scheme-dependent series expansions in powers of $\alpha_{\text {IR }}$ [75-76] (see also [77]).

\section{BARYON OPERATORS}

In this section we consider a theory with gauge group $G=\mathrm{SU}(3)$ and $N_{f}$ fermions in the fundamental (triplet) representation, $R=F$. Since the fermions are massless, the ultraviolet theory is invariant under the global flavor $(f l$.) symmetry group

$$
G_{f l .}=\mathrm{SU}\left(N_{f}\right)_{L} \otimes \mathrm{SU}\left(N_{f}\right)_{R} \otimes \mathrm{U}(1)_{V} .
$$

This symmetry is unbroken in the non-Abelian Coulomb phase. Hence, the baryon operators that we consider transform according to definite representations of this group. Each fermion field can be decomposed into its left- and righthanded chiral components as $\psi=\left(P_{L}+P_{R}\right) \psi=\psi_{L}+\psi_{R}$, where $P_{R, L}=(1 / 2)\left(1 \pm \gamma_{5}\right)$ and we suppress color and flavor indices here. Showing these latter indices explicitly, each fermion field can thus be written formally as $\psi_{i, L}^{a}+\psi_{i, R}^{a}$, where $a$ is an $\mathrm{SU}(3)$ color gauge index. Here, the flavor index $i$ on $\psi_{i, L}^{a}$ refers to the fundamental representation of $\mathrm{SU}\left(N_{f}\right)_{L}$, while the flavor index $i$ on $\psi_{i, R}^{a}$ refers to the fundamental representation of $\mathrm{SU}\left(N_{f}\right)_{R}$. This will be understood implicitly below. The chiral components $\psi_{i, L}^{a}$ and $\psi_{i, R}^{a}$ transform as $\left(N_{f}, 1\right)$ and $\left(1, N_{f}\right)$ under the chiral part of $G_{f l}, \mathrm{SU}\left(N_{f}\right)_{L} \otimes \mathrm{SU}\left(N_{f}\right)_{R}$. The bilinear operator $\bar{\psi} \psi=$ $\sum_{i=1}^{N_{f}}\left(\bar{\psi}_{i, L} \psi_{i, R}+\bar{\psi}_{i, R} \psi_{i, L}\right)$ thus corresponds to what would be the flavor-singlet in the confined phase, where the chiral part of $G_{f l}$. is broken to the diagonal $\mathrm{SU}\left(N_{f}\right)_{V}$ subgroup, while the operator $\bar{\psi} \mathcal{T}_{j} \psi$ corresponds to what would be the flavor-adjoint in the confined phase. In our present work we will use the symbols $S_{k, L}$ and $A_{k, L}$ to denote the $k$-fold symmetric and $k$-fold antisymmetric representations of $\mathrm{SU}\left(N_{f}\right)_{L}$, and similarly with $S_{k, R}$ and $A_{k, R}$ with $\mathrm{SU}\left(N_{f}\right)_{R}$.

Clearly, all of our baryon operators have unit baryonic charge under the $\mathrm{U}(1)_{V}$ factor group [which is equivalent to $\mathrm{U}(1)_{B}$ here] so we leave this implicit henceforth. Although we are in an NACP without any confinement of color, it is nonetheless convenient to deal with gauge-singlet operators, since they are gauge-invariant. The invariance of the baryon operator under the $\mathrm{SU}(3)$ gauge group is guaranteed by the contraction of the color indices $a, b, c$ on the three fermion fields with the $\epsilon_{a b c}$ tensor, so that the color part of the baryon wavefunction is totally antisymmetric. The other parts of the baryon operator depend on the chirality, spin contractions, and flavor structure of the three-fermion operator. These are constrained by the requirement that the full wavefunction must be totally antisymmetric under interchange of any two of the fermions.

As is well known, relevant representations of the Lorentz group $\mathrm{SO}(3,1)$ are specified by two spins, $\left(j_{1}, j_{2}\right)$. It is convenient to construct a subset of baryon operators by combining two of the three fermions in a Majorana-type bilinear operator product, since this has spin 0 and is Lorentz-invariant. A Majorana-type bilinear links lefthanded to left-handed chiral components of a fermion, and right-handed to right-handed chiral components. There are thus two of these, namely $\psi_{i, L}^{a T} C \psi_{j, L}^{b}$ and $\psi_{i, R}^{a T} \psi_{j, R}^{b}$. Here, $C$ is the Dirac charge conjugation matrix defined by $C \gamma_{\mu} C^{-1}=-\gamma_{\mu}^{T}$ and satisfying the properties $C^{T}=-C$ and $C^{-1}=C^{T}$. The full baryon operator product is then obtained by combining each of these Majorana-type bilinears with the left-handed or right-handed chiral fermion. One thus has the operators

$$
\begin{aligned}
& \mathcal{O}_{R L L}=\epsilon_{a b c} \psi_{i, R}^{a}\left[\psi_{j, L}^{b T} C \psi_{k, L}^{c}\right] \\
& \mathcal{O}_{L R R}=\epsilon_{a b c} \psi_{i, L}^{a}\left[\psi_{j, R}^{b T} C \psi_{k, R}^{c}\right] \\
& \mathcal{O}_{R R R}=\epsilon_{a b c} \psi_{i, R}^{a}\left[\psi_{j, R}^{b T} C \psi_{k, R}^{c}\right]
\end{aligned}
$$

and

$$
\mathcal{O}_{L L L}=\epsilon_{a b c} \psi_{i, L}^{a}\left[\psi_{j, L}^{b T} C \psi_{k, L}^{c}\right]
$$

To distinguish the chirality of the unpaired fermion, one could use a subscript $L$ or $R$, but we shall follow the notational conventions of $[55,58]$, according to which

$$
\mathcal{O}_{+}^{\left(\frac{1}{2}, 0\right)} \equiv \mathcal{O}_{L L L}^{\left(\frac{1}{2}, 0\right)}
$$

and 


$$
\mathcal{O}_{-}^{\left(\frac{1}{2}, 0\right)} \equiv \mathcal{O}_{R L L}^{\left(\frac{1}{2}, 0\right)}
$$

As is evident, in the Lorentz $\left(j_{1}, j_{2}\right)$ labeling, the $j_{1}=1 / 2$ refers to the fermion field that is not a member of the Majorana fermion bilinear, and $j_{2}=0$ refers to the spin-0 transformation property of this Majorana fermion bilinear. These operators have anomalous dimensions denoted $\gamma_{B}^{\left(\frac{1}{2}, 0\right),+}$ and $\gamma_{B}^{\left(\frac{1}{2}, 0\right),-}$, respectively. Because the theory at the IRFP in the non-Abelian phase preserves the full flavor symmetry (2.1), the anomalous dimension $\gamma_{B}^{\left(\frac{1}{2}, 0\right),+}$ for $\mathcal{O}_{L L L}^{\left(\frac{1}{2}, 0\right)}$ is equal to the anomalous dimension for the corresponding operator with all $L$ indices switched to $R$, namely $\mathcal{O}_{R R R}^{\left(\frac{1}{2}, 0\right)}$, and, separately, the anomalous dimension $\gamma_{B}^{\left(\frac{1}{2}, 0\right),-}$ for $\mathcal{O}_{R L L}^{\left(\frac{1}{2}, 0\right)}$ is equal to the anomalous dimension for the corresponding operator with $L$ and $R$ indices interchanged, namely $\mathcal{O}_{L R R}^{\left(\frac{1}{2}, 0\right)}$.

One part of the classification of baryon operators entails the analysis of the combination of the three spin $1 / 2$ representations of angular momentum SU(2). In general, one has

$$
\frac{1}{2} \times \frac{1}{2} \times \frac{1}{2}=\frac{1}{2}+\frac{1}{2}+\frac{3}{2}
$$

(i.e., $2 \times 2 \times 2=2+2+4$ in terms of the dimensions $2 s+1$ of the representations). We have considered above the cases in which two of the spins are contracted to produce spin 0 , corresponding to one of the two spin- $1 / 2$ terms on the right-hand side of Eq. (2.8). There are two remaining cases to consider, in which one combines two of the spins to produce a spin- 1 state and then combines this with the third spin $1 / 2$ to yield a net spin $1 / 2$ or spin $3 / 2$. We recall that the spin wavefunction in the case of spin $3 / 2$ is totally symmetric, i.e., $S_{3}$ under the $\mathrm{SU}(2)$ of spin. In the analysis of baryon operators in QCD, it has proved useful to introduce a vector $\Delta_{\mu}$ that is lightlike, i.e., has the property $\Delta^{2}=0$, and consider the operators (leaving the flavor indices implicit)

$$
\begin{aligned}
& \mathcal{O}_{L L L}^{\left(\frac{3}{2}, 0\right)}=\epsilon_{a b c} \Delta \psi_{L}^{a} \Delta \psi_{L}^{b} \Delta \psi_{L}^{c} \\
& \mathcal{O}_{R R R}^{\left(\frac{3}{2}, 0\right)}=\epsilon_{a b c} \Delta \psi_{R}^{a} \Delta \psi \psi_{R}^{b} \Delta \psi_{R}^{c} \\
& \mathcal{O}_{L L R}^{\left(1, \frac{1}{2}\right)}=\epsilon_{a b c} \Delta \psi_{L}^{a} \Delta \psi \psi_{L}^{b} \Delta \psi_{R}^{c}
\end{aligned}
$$

and

$$
\mathcal{O}_{R R L}^{\left(1, \frac{1}{2}\right)}=\epsilon_{a b c} \phi \psi_{R}^{a} \phi \psi \psi_{R}^{b} \phi \psi_{L}^{c} .
$$

In the notation of $[55,58]$,

$$
\mathcal{O}_{+}^{\left(\frac{3}{2}, 0\right)} \equiv \mathcal{O}_{L L L}^{\left(\frac{3}{2}, 0\right)}
$$

and

$$
\mathcal{O}_{-}^{\left(1, \frac{1}{2}\right)} \equiv \mathcal{O}_{L L R}^{\left(1, \frac{1}{2}\right)}
$$

The anomalous dimensions of these operators are denoted $\gamma^{\left(\frac{3}{2}, 0\right),+}$ and $\gamma^{\left(1, \frac{1}{2}\right),-}$, respectively. Again, owing to the exact chiral symmetry (2.1), the anomalous dimension $\gamma^{\left(\frac{3}{2}, 0\right),+}$ of $\mathcal{O}_{L L L}^{\left(\frac{3}{2}, 0\right)}$ is equal to the anomalous dimension of $\mathcal{O}_{R R R}^{\left(\frac{3}{2}, 0\right)}$, and the anomalous dimension $\gamma^{\left(1, \frac{1}{2}\right),-}$ of $\mathcal{O}_{L L R}^{\left(1, \frac{1}{2}\right)}$ is equal to the anomalous dimension of $\mathcal{O}_{R R L}^{\left(1, \frac{1}{2}\right)}$. The normalization of these anomalous dimensions is fixed by the basic relation (1.1).

\section{SCHEME-INDEPENDENT SERIES EXPANSION FOR ANOMALOUS DIMENSION OF GENERAL BARYON OPERATOR}

A general expression, calculated to the two-loop level, was given for the anomalous dimension of a general baryon operator $\mathcal{O}_{B}$ in [57] and extended to the three-loop level in $[58,59]$. This depends on certain coefficients $\mathbb{C}_{k}$, which are listed in Table I. With the definition (1.1) (which sets the absolute normalization of the anomalous dimension), and noting that the sign convention in (1.1) is opposite to that in [58], we have

$$
\begin{aligned}
\gamma_{B}= & \frac{1}{3} \mathbb{C}_{2} a+\left[\left(-72+4 N_{f}\right) \mathbb{C}_{0}+\left(\frac{47}{18}-\frac{1}{27} N_{f}\right) \mathbb{C}_{2}+\frac{1}{36} \mathbb{C}_{2}^{2}-\frac{5}{36} \mathbb{C}_{4}\right] a^{2} \\
& +\left[\left(-\frac{16094}{9}-34 \zeta_{3}+\frac{1706}{9} N_{f}-\frac{20}{9} N_{f}^{2}\right) \mathbb{C}_{0}+\left(\frac{5873}{108}-\frac{433}{18} \zeta_{3}-\left(\frac{71}{27}+\frac{40}{9} \zeta_{3}\right) N_{f}-\frac{13}{81} N_{f}^{2}\right) \mathbb{C}_{2}\right. \\
& +\left(-\frac{209}{324}+\frac{71}{27} \zeta_{3}+\frac{1}{324} N_{f}\right) \mathbb{C}_{2}^{2}+\left(\frac{5}{648}-\frac{1}{27} \zeta_{3}\right) \mathbb{C}_{2}^{3}+\left(\frac{91}{72}-\frac{29}{12} \zeta_{3}+\frac{7}{324} N_{f}\right) \mathbb{C}_{4} \\
& \left.+\left(-\frac{37}{432}+\frac{25}{144} \zeta_{3}\right) \mathbb{C}_{2} \mathbb{C}_{4}+\left(-\frac{1}{8}+\frac{2}{9} \zeta_{3}\right) \mathbb{C}_{444}\right] a^{3}+O\left(a^{4}\right) .
\end{aligned}
$$


TABLE I. Values of $\mathbb{C}_{k}$ coefficients.

\begin{tabular}{lccccc}
\hline \hline$\left(j_{1}, j_{2}\right)$ & chirality & $\mathbb{C}_{0}$ & $\mathbb{C}_{2}$ & $\mathbb{C}_{4}$ & $\mathbb{C}_{444}$ \\
\hline$\left(\frac{1}{2}, 0\right)$ & + & 1 & 12 & 72 & 0 \\
$\left(\frac{1}{2}, 0\right)$ & - & 1 & 12 & -24 & 0 \\
$\left(\frac{3}{2}, 0\right)$ & + & 1 & -12 & 72 & 0 \\
$\left(1, \frac{1}{2}\right)$ & - & 1 & -4 & -24 & 0 \\
\hline \hline
\end{tabular}

We list the values of the $\mathbb{C}_{k}$ coefficients for various specific baryon operators in Table I.

We denote the anomalous dimension of the general baryon operator $\mathcal{O}_{B}$ as $\gamma_{\mathcal{O}_{B}}$ and write the scheme-independent series expansion for this as

$$
\gamma_{B}=\sum_{n=1}^{\infty} \kappa_{B, n} \Delta_{f}^{n}
$$

For this $\mathrm{SU}(3)$ gauge theory with $N_{f}$ fermions in the fundamental representation, $N_{u}=33 / 2$, so the general expression for $\Delta_{f}$ in Eq. (1.6) yields $\Delta_{f}=(33 / 2)-N_{f}$.

We calculate the following coefficients in this schemeindependent series expansion for the general baryon operator:

$$
\kappa_{B, 1}=\frac{2}{3^{2} \cdot(107)} \mathbb{C}_{2},
$$

$$
\begin{aligned}
\kappa_{B, 2}= & -\frac{8}{3 \cdot(107)^{2}} \mathbb{C}_{0}+\frac{27083}{2 \cdot 3^{4} \cdot(107)^{3}} \mathbb{C}_{2} \\
& +\frac{1}{3^{4} \cdot(107)^{2}}\left(\mathbb{C}_{2}^{2}-5 \mathbb{C}_{4}\right)
\end{aligned}
$$

and

$$
\begin{aligned}
\kappa_{B, 3}= & \left(\frac{291892}{3^{5} \cdot(107)^{4}}-\frac{272}{3^{3} \cdot(107)^{3}} \zeta_{3}\right) \mathbb{C}_{0}+\left(\frac{352124197}{2^{2} \cdot 3^{6} \cdot(107)^{5}}-\frac{238124}{3^{5} \cdot(107)^{4}} \zeta_{3}\right) \mathbb{C}_{2} \\
& +\left(-\frac{47365}{2 \cdot 3^{7} \cdot(107)^{4}}+\frac{568}{3^{6} \cdot(107)^{3}} \zeta_{3}\right) \mathbb{C}_{2}^{2}+\left(\frac{16525}{2 \cdot 3^{6} \cdot(107)^{4}}-\frac{58}{3^{4} \cdot(107)^{3}} \zeta_{3}\right) \mathbb{C}_{4} \\
& +\left(\frac{5}{3^{7} \cdot(107)^{3}}-\frac{8}{3^{6} \cdot(107)^{3}} \zeta_{3}\right) \mathbb{C}_{2}^{3}+\left(-\frac{37}{2 \cdot 3^{6} \cdot(107)^{3}}+\frac{25}{2 \cdot 3^{5} \cdot(107)^{3}} \zeta_{3}\right) \mathbb{C}_{2} \mathbb{C}_{4} \\
& +\left(-\frac{1}{3^{3} \cdot(107)^{3}}+\frac{16}{3^{5} \cdot(107)^{3}} \zeta_{3}\right) \mathbb{C}_{444},
\end{aligned}
$$

where $\zeta_{s}=\sum_{n=1}^{\infty} n^{-s}$ is the Riemann zeta function. In Eqs. (3.3)-(3.5) we have indicated the simple factorizations of the denominators. The numerators do not, in general, have such simple factorizations.

In floating-point format, to the indicated precision,

$$
\kappa_{B, 1}=\left(2.076843 \times 10^{-3}\right) \mathbb{C}_{2},
$$

$$
\begin{aligned}
\kappa_{B, 2}= & -\left(2.329170 \times 10^{-4}\right) \mathbb{C}_{0}+\left(1.364679 \times 10^{-4}\right) \mathbb{C}_{2} \\
& +\left(1.078319 \times 10^{-6}\right) \mathbb{C}_{2}^{2}-\left(5.391597 \times 10^{-6}\right) \mathbb{C}_{4},
\end{aligned}
$$

and

$$
\begin{aligned}
\kappa_{B, 3}= & -\left(0.721139 \times 10^{-6}\right) \mathbb{C}_{0}-\left(0.376693 \times 10^{-6}\right) \mathbb{C}_{2} \\
& +\left(0.681918 \times 10^{-6}\right) \mathbb{C}_{2}^{2}-\left(0.616147 \times 10^{-6}\right) \mathbb{C}_{4} \\
& -\left(0.890178 \times 10^{-8}\right) \mathbb{C}_{2}^{3}+\left(2.975975 \times 10^{-8}\right) \mathbb{C}_{2} \mathbb{C}_{4} \\
& +\left(0.343749 \times 10^{-7}\right) \mathbb{C}_{444} .
\end{aligned}
$$

\section{SCHEME-INDEPENDENT SERIES EXPANSIONS FOR ANOMALOUS DIMENSIONS OF SPECIFIC BARYON OPERATORS}

In this section we present results for coefficients in scheme-independent series expansions for the anomalous dimensions of specific baryon operators. These analytic results are new here. The anomalous dimension of the baryon operator $\mathcal{O}_{ \pm}^{\left(j_{1}, j_{2}\right)}$ is denoted $\gamma_{B}^{\left(j_{1}, j_{2}\right), \pm}$. We express the scheme-independent series expansion for this anomalous dimension as

$$
\gamma_{B}^{\left(j_{1}, j_{2}\right), \pm}=\sum_{n=1}^{\infty} \kappa_{n}^{\left(j_{1}, j_{2}\right), \pm} \Delta_{f}^{n}
$$

The truncation of this infinite series to maximal power (order) $\Delta_{f}^{p}$ is denoted $\gamma_{B, \Delta_{f}^{p}}^{\left(j_{1}, j_{2}\right), \pm}$. We note that numerical results for the $\Delta_{f}$ series expansions for two of the four specific operators, namely, $\mathcal{O}_{ \pm}^{\left(\frac{1}{2}, 0\right)}$, were given previously in [60]. Since they were based on the results of [58], they should be multiplied by a factor of 2 [59]. 
We calculate the following:

$$
\begin{aligned}
\kappa_{1}^{\left(\frac{1}{2}, 0\right),+} & =\frac{8}{3 \cdot(107)}=2.492212 \times 10^{-2} \\
\kappa_{2}^{\left(\frac{1}{2}, 0\right),+} & =\frac{38758}{3^{3} \cdot(107)^{3}}=1.171780 \times 10^{-2} \\
\kappa_{3}^{\left(\frac{1}{2}, 0\right),+} & =\frac{314021069}{3^{5} \cdot(107)^{5}}-\frac{97792}{3^{3} \cdot(107)^{4}} \zeta_{3} \\
& =5.892227 \times 10^{-5}
\end{aligned}
$$$$
\kappa_{1}^{\left(\frac{1}{2}, 0\right),-}=\frac{8}{3 \cdot(107)}=2.492212 \times 10^{-2}
$$$$
\kappa_{2}^{\left(\frac{1}{2}, 0\right),-}=\frac{18626}{3^{2} \cdot(107)^{3}}=1.689374 \times 10^{-3}
$$$$
\kappa_{3}^{\left(\frac{1}{2}, 0\right),-}=\frac{40784885}{3^{3} \cdot(107)^{5}}-\frac{70400}{3^{3} \cdot(107)^{4}} \zeta_{3}
$$$$
=0.837892 \times 10^{-4}
$$$$
\kappa_{1}^{\left(\frac{3}{2}, 0\right),+}=-\frac{8}{3 \cdot(107)}=-\left(2.492212 \times 10^{-2}\right)
$$$$
\kappa_{2}^{\left(\frac{3}{2}, 0\right),+}=-\frac{69574}{3^{3} \cdot(107)^{3}}=-\left(2.103448 \times 10^{-3}\right)
$$$$
\kappa_{3}^{\left(\frac{3}{2}, 0\right),+}=-\frac{32245429}{3^{3} \cdot(107)^{5}}+\frac{1169920}{3^{4} \cdot(107)^{4}} \zeta_{3}
$$$$
=4.730261 \times 10^{-5}
$$$$
\kappa_{1}^{\left(1, \frac{1}{2}\right),-}=-\frac{8}{3^{2} \cdot(107)}=-\left(0.830737 \times 10^{-2}\right)
$$$$
\kappa_{2}^{\left(1, \frac{1}{2}\right),-}=-\frac{62726}{3^{4} \cdot(107)^{3}}=-\left(6.321370 \times 10^{-4}\right)
$$$$
\kappa_{3}^{\left(1, \frac{1}{2}\right),-}=-\frac{314714429}{3^{6} \cdot(107)^{5}}+\frac{178688}{3^{3} \cdot(107)^{4}} \zeta_{3}
$$$$
=2.991050 \times 10^{-5} \text {. }
$$

As is evident from these results, all of the schemeindependent coefficients $\kappa_{n}^{\left(\frac{1}{2}, 0\right),+}$ and $\kappa_{n}^{\left(\frac{1}{2}, 0\right),-}$ that have been calculated, namely those for $n=1,2,3$, are positive. In contrast, we find mixed signs for the scheme-independent coefficients $\kappa_{n}^{\left(\frac{3}{2}, 0\right),+}$; while $\kappa_{1}^{\left(\frac{3}{2}, 0\right),+}$ and $\kappa_{2}^{\left(\frac{3}{2}, 0\right),+}$ are negative, $\kappa_{3}^{\left(\frac{3}{2}, 0\right),+}$ is positive, and similarly with the $\kappa_{n}^{\left(1, \frac{1}{2}\right),-}$ for $n=1$, 2,3 .

In Figs. 1-4 we show curves of these anomalous dimensions, and in Tables II-V we list values of these

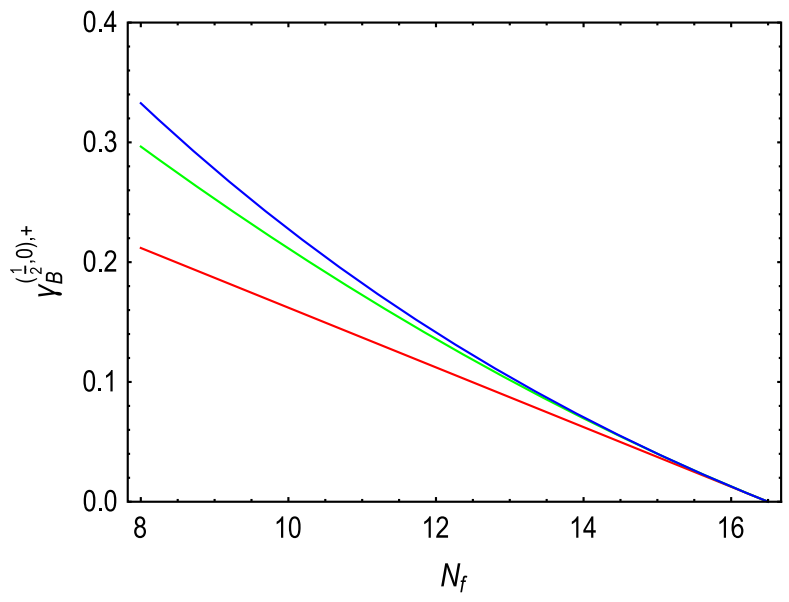

FIG. 1. Plot of $\gamma_{B}^{\left(\frac{1}{2}, 0\right),+}$, as calculated in the scheme-independent series expansion to $O\left(\Delta_{f}^{p}\right)$ with $1 \leq p \leq 3$, as a function of $N_{f}$. The curves refer to the calculation to $O\left(\Delta_{f}\right)$ (red); $O\left(\Delta_{f}^{2}\right)$ (green); and $O\left(\Delta_{f}^{3}\right)$ (blue), with colors online.

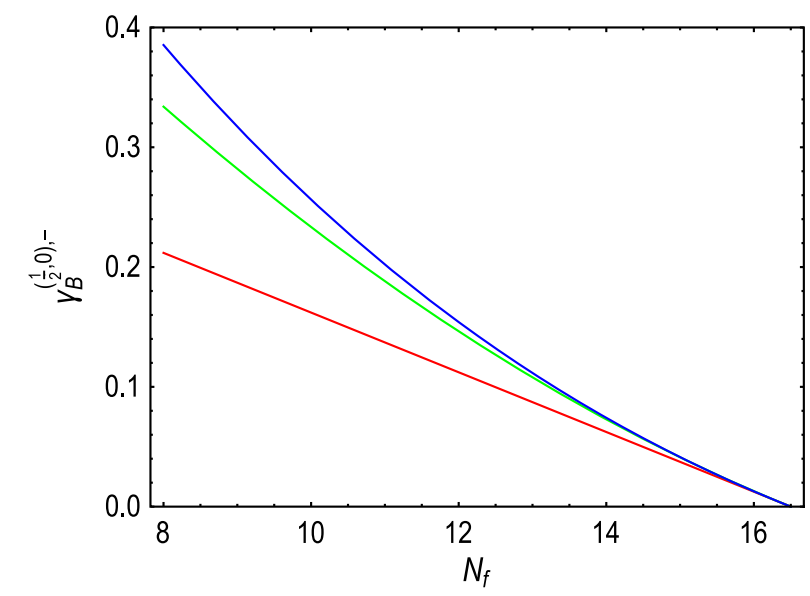

FIG. 2. Plot of $\gamma_{B}^{\left(\frac{1}{2}, 0\right),-}$, as calculated in the scheme-independent series expansion to $O\left(\Delta_{f}^{p}\right)$ with $1 \leq p \leq 3$, as a function of $N_{f}$. The curves refer to the calculation to $O\left(\Delta_{f}\right)$ (red); $O\left(\Delta_{f}^{2}\right)$ (green); and $O\left(\Delta_{f}^{3}\right)$ (blue), with colors online.

anomalous dimensions, as calculated to the various orders in $\Delta_{f}$ in our scheme-independent expansions.

We comment further on the results for the coefficients $\kappa_{n}^{\left(\frac{1}{2}, 0\right),+}$ and $\kappa_{n}^{\left(\frac{1}{2}, 0\right),-}$ in the respective scheme-independent series expansions for $\gamma_{B}^{\left(\frac{1}{2}, 0\right), \pm}$. It will be recalled that an important property of the scheme-independent calculations of $\gamma_{\bar{\psi} \psi, \text { IR }}$ in [33-39] is that (a) the coefficients $\kappa_{1}$ and $\kappa_{2}$ are manifestly positive, and (b) for all groups and representations considered, $\kappa_{3}$ and $\kappa_{4}$ were also found to be positive. This result implied several monotonicity properties, namely that (i) for a fixed truncation order $p$, the schemeindependent series expansion for $\gamma_{\bar{\psi} \psi, \mathrm{IR}}$ is a monotonically increasing function of $\Delta_{f}$, i.e., it increases monotonically with decreasing $N_{f}$, and (ii) for a fixed value of $N_{f}$, the 


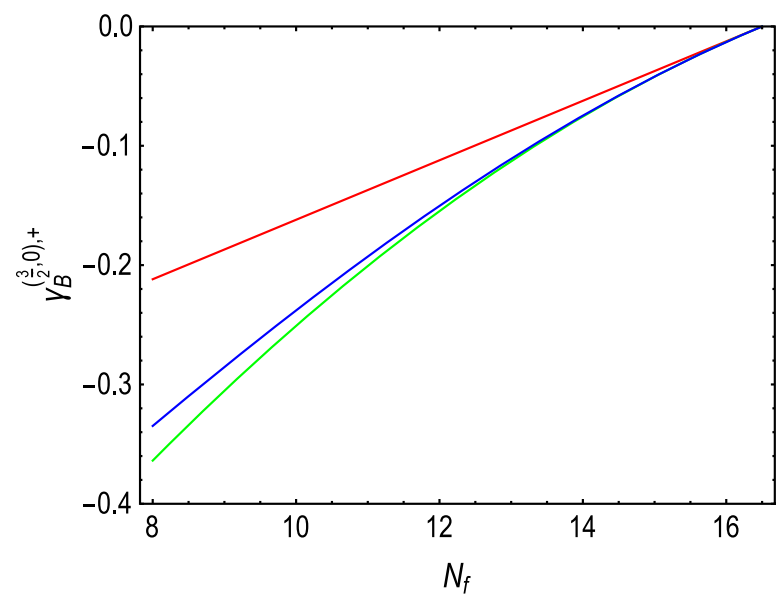

FIG. 3. Plot of $\gamma_{B}^{\left(\frac{3}{2}, 0\right),+}$, as calculated in the scheme-independent series expansion to $O\left(\Delta_{f}^{p}\right)$ with $1 \leq p \leq 3$, as a function of $N_{f}$. The curves refer to the calculation to $O\left(\Delta_{f}\right)$ (red); $O\left(\Delta_{f}^{2}\right)$ (green); and $O\left(\Delta_{f}^{3}\right)$ (blue), with colors online.

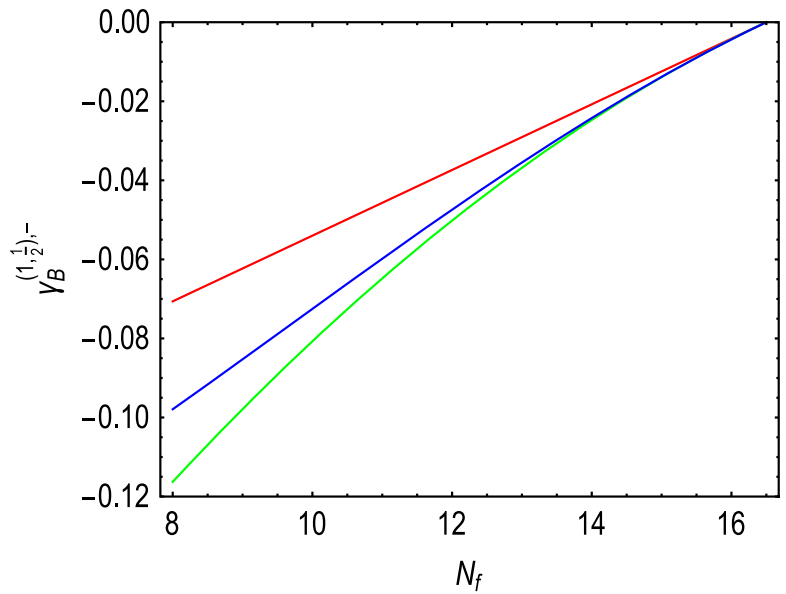

FIG. 4. Plot of $\gamma_{B}^{\left(1, \frac{1}{2}\right),-}$, as calculated in the scheme-independent series expansion to $O\left(\Delta_{f}^{p}\right)$ with $1 \leq p \leq 3$, as a function of $N_{f}$. The curves refer to the calculation to $O\left(\Delta_{f}\right)$ (red); $O\left(\Delta_{f}^{2}\right)$ (green); and $O\left(\Delta_{f}^{3}\right)$ (blue), with colors online.

series calculation to $O\left(\Delta_{f}^{p}\right)$ is a monotonically increasing function of $p$. Indeed, as was noted in several of these works, and was studied in detail in [39], the coefficients in the corresponding scheme-independent expansions of anomalous dimensions of composite holomorphic products of chiral superfields in $\mathcal{N}=1$ supersymmetric gauge theories are all positive.

In view of these previous positivity findings, it is of considerable interest that all of the $\kappa_{n}^{\left(\frac{1}{2}, 0\right),+}$ and $\kappa_{n}^{\left(\frac{1}{2}, 0\right),-}$ that have been calculated, namely those for $j=1,2,3$, are positive, so the corresponding monotonicity results apply for $\gamma_{B}^{\left(\frac{1}{2}, 0\right), \pm}$. These calculations to finite order in $O\left(\Delta_{f}\right)$ are expected to be most accurate for small $\Delta_{f}$, i.e., for $N_{f}$
TABLE II. Values of $\gamma_{B, \Delta^{p}}^{\left(\frac{1}{2}, 0\right),+}$ with $1 \leq p \leq 3$.

\begin{tabular}{llll}
\hline \hline$N_{f}$ & $\gamma_{B, \Delta^{1}}^{\left(\frac{1}{2}, 0\right)+}$ & $\gamma_{B, \Delta^{2}}^{\left(\frac{1}{2}, 0\right)+}$ & $\gamma_{B, \Delta^{3}}^{\left(\frac{1}{2}, 0\right),+}$ \\
\hline 8 & 0.212 & 0.296 & 0.333 \\
9 & 0.187 & 0.253 & 0.278 \\
10 & 0.162 & 0.212 & 0.228 \\
11 & 0.137 & 0.173 & 0.182 \\
12 & 0.112 & 0.136 & 0.141 \\
13 & 0.0872 & 0.102 & 0.104 \\
14 & 0.0623 & 0.0696 & 0.0705 \\
15 & 0.0374 & 0.0400 & 0.0402 \\
16 & 0.0125 & 0.0128 & 0.0128 \\
\hline \hline
\end{tabular}

TABLE III. Values of $\gamma_{B, \Delta^{p}}^{\left(\frac{1}{2}, 0\right),-}$ with $1 \leq p \leq 3$.

\begin{tabular}{llll}
\hline \hline$N_{f}$ & $\gamma_{B, \Delta^{1}}^{\left(\frac{1}{2}, 0\right),-}$ & $\gamma_{B, \Delta^{2}}^{\left(\frac{1}{2}, 0\right),-}$ & $\gamma_{B, \Delta^{3}}^{\left(\frac{1}{2}, 0\right),-}$ \\
\hline 8 & 0.212 & 0.334 & 0.385 \\
9 & 0.187 & 0.282 & 0.317 \\
10 & 0.162 & 0.233 & 0.256 \\
11 & 0.137 & 0.188 & 0.202 \\
12 & 0.112 & 0.146 & 0.154 \\
13 & 0.0872 & 0.108 & 0.112 \\
14 & 0.0623 & 0.0729 & 0.0742 \\
15 & 0.0374 & 0.0412 & 0.0415 \\
16 & 0.0125 & 0.0129 & 0.0129 \\
\hline \hline
\end{tabular}

TABLE IV. Values of $\gamma_{B, \Delta^{p}}^{\left(\frac{3}{2}, 0\right),+}$ with $1 \leq p \leq 3$.

\begin{tabular}{llll}
\hline \hline$N_{f}$ & $\gamma_{B, \Delta^{1}}^{\left(\frac{3}{2}, 0\right),+}$ & $\gamma_{B, \Delta^{2}}^{\left(\frac{3}{2}, 0\right),+}$ & $\gamma_{B, \Delta^{3}}^{\left(\frac{3}{2}, 0\right),+}$ \\
\hline 8 & -0.212 & -0.364 & -0.335 \\
9 & -0.187 & -0.305 & -0.285 \\
10 & -0.162 & -0.251 & -0.238 \\
11 & -0.137 & -0.201 & -0.193 \\
12 & -0.112 & -0.155 & -0.150 \\
13 & -0.0872 & -0.113 & -0.111 \\
14 & -0.0623 & -0.0755 & -0.0747 \\
15 & -0.0374 & -0.0421 & -0.0420 \\
16 & -0.0125 & -0.0130 & -0.0130 \\
\hline \hline
\end{tabular}

TABLE V. Values of $\gamma_{B, \Delta^{p}}^{\left(1, \frac{1}{2}\right),-}$ with $1 \leq p \leq 3$.

\begin{tabular}{llll}
\hline \hline$N_{f}$ & $\gamma_{B, \Delta^{1}}^{\left(1, \frac{1}{2}\right),-}$ & $\gamma_{B, \Delta^{2}}^{\left(1, \frac{1}{2}\right),-}$ & $\gamma_{B, \Delta^{3}}^{\left(1, \frac{1}{2}\right),-}$ \\
\hline 8 & -0.0706 & -0.117 & -0.0979 \\
9 & -0.0623 & -0.0979 & -0.0852 \\
10 & -0.0540 & -0.0807 & -0.0725 \\
11 & -0.0457 & -0.0648 & -0.0598 \\
12 & -0.0374 & -0.0502 & -0.0475 \\
13 & -0.0291 & -0.0368 & -0.0355 \\
14 & -0.0208 & -0.0247 & -0.0243 \\
15 & -0.0125 & -0.0139 & -0.0138 \\
16 & -0.00415 & -0.00431 & -0.00431 \\
\hline \hline
\end{tabular}


slightly below $N_{u}=16.5$, while higher-order corrections become progressively larger as $N_{f}$ decreases toward the lower end of the NACP. In [34-37] these schemeindependent calculations were used to derive estimates of the value of $N_{f}$ at the lower end of the NACP. The method was to use the unitarity lower bound $D_{\mathcal{O}} \geq 1$ for a Lorentz-scalar operator $\mathcal{O}$ in a conformal field theory [49]. From the basic definition (1.1), taking into account that the free-field (classical) dimension of $\bar{\psi} \psi$ is $D_{\bar{\psi} \psi, c l}=3$, there follows the upper bound $\gamma_{\bar{\psi} \mu, \mathrm{IR}} \leq 2$. Combining this with the above-mentioned monotonicity results for the schemeindependent calculation of $\gamma_{\bar{\psi} \psi}, \mathrm{IR}$ yielded the estimate [34-37] that the conformal non-Abelian Coulomb phase extends from $N_{u}=16.5$ down to slightly above $N_{f}=8$, so the maximal value of $\Delta_{f}$ in this NACP, is $\left(\Delta_{f}\right)_{\max } \simeq 8$.

As was done for $\gamma_{\bar{\psi} \psi, \mathrm{IR}}$ and $\beta_{\mathrm{IR}}^{\prime}$ in previous works [33-35,37], we may estimate the accuracy of these $O\left(\Delta_{f}^{3}\right)$ series calculations of $\gamma_{B}^{\left(\frac{1}{2}, 0\right),+}$ and $\gamma_{B}^{\left(\frac{1}{2}, 0\right),-}$ in several ways. The first is to plot the various truncations to $O\left(\Delta_{f}^{p}\right)$ with $p=1,2,3$ as functions of $\Delta_{f}$, or equivalently, $N_{f}$ in the conformal regime (non-Abelian Coulomb phase) and ascertain how close the curves are to each other. As expected, the curves of $\gamma_{B}^{\left(\frac{1}{2}, 0\right),+}$, calculated to the higher two orders, $O\left(\Delta_{f}^{2}\right)$ and $O\left(\Delta_{f}^{3}\right)$, remain close to each other over a larger range, extending to lower $N_{f}$, than the corresponding curves calculated to the lower two orders, $O\left(\Delta_{f}\right)$ and $O\left(\Delta_{f}^{2}\right)$. A similar comment applies to the corresponding curves of $\gamma_{B}^{\left(\frac{1}{2}, 0\right),-}$.

We recall that if a function $f(z)$ is analytic at $z=0$ and thus has a Taylor series $f(z)=\sum_{n=1}^{\infty} s_{n} z^{n}$, then the ratio test states that the series converges to the function $f(z)$ if $|z|<z_{0}$, where

$$
z_{0}=\lim _{n \rightarrow \infty} \frac{\left|s_{n}\right|}{\left|s_{n+1}\right|} .
$$

Of course, even if these series expansions in powers of $\Delta_{f}$ were Taylor series, it would not be possible to actually calculate the limit (4.14), since we have only the first few coefficients. Furthermore, the $\Delta_{f}$ expansion is not generically expected to be a Taylor series, because the properties of the theory change qualitatively as $N_{f}$ increases through $N_{u}$ and the theory becomes IR-free instead of UV-free. Nevertheless, a calculation of the first few ratios can give a rough idea of the accuracy of a truncation of the series to a given order. Accordingly, this was carried out for $\gamma_{\bar{\psi} \psi, \text { IR }}$ and $\beta_{\mathrm{IR}}^{\prime}$ in [33-38]. It was found that the series expansions for $\gamma_{\bar{\psi} \psi, \mathrm{IR}}$ to $O\left(\Delta_{f}^{4}\right)$ and $\beta_{\mathrm{IR}}^{\prime}$ to $O\left(\Delta_{f}^{5}\right)$ were reasonably accurate over a substantial portion of the NACP.

It is thus worthwhile to carry out the analogous calculation of ratios here for $\gamma_{B}^{\left(\frac{1}{2}, 0\right), \pm}$. We find

$$
\begin{aligned}
& \frac{\kappa_{1}^{\left(\frac{1}{2}, 0\right),+}}{\kappa_{2}^{\left(\frac{1}{2}, 0\right),+}}=21.27 \\
& \frac{\kappa_{2}^{\left(\frac{1}{2}, 0\right),+}}{\kappa_{3}^{\left(\frac{1}{2}, 0\right),+}}=19.89 \\
& \frac{\kappa_{1}^{\left(\frac{1}{2}, 0\right),-}}{\kappa_{2}^{\left(\frac{1}{2}, 0\right),-}}=14.75
\end{aligned}
$$

and

$$
\frac{\kappa_{2}^{\left(\frac{1}{2}, 0\right),-}}{\kappa_{3}^{\left(\frac{1}{2}, 0\right),-}}=20.16 .
$$

These ratios are all substantially larger than $\left(\Delta_{f}\right)_{\max } \simeq 8$, indicating that the scheme-independent series expansions for $\gamma_{B}^{\left(\frac{1}{2}, 0\right), \pm}$ to $O\left(\Delta_{f}^{3}\right)$ may be reasonably accurate over a substantial part of the NACP for this SU(3) theory.

\section{UNITARITY BOUNDS ON ANOMALOUS DIMENSIONS OF BARYONIC OPERATORS}

Since our scheme-independent series expansions for baryon operators apply at an infrared fixed point in the non-Abelian Coulomb phase, where the theory is conformally invariant, it is of interest to study how the resultant anomalous dimensions compare with the unitarity bounds on a conformal field theory. In general [49], for an operator $\mathcal{O}$ characterized by Lorentz spins $\left(j_{1}, j_{2}\right)$, unitarity in a conformal field theory requires that the full scaling dimension $D_{\mathcal{O}}$ is bounded below according to

$$
D_{\mathcal{O}} \geq j_{1}+j_{2}+1 .
$$

For our case of $\mathrm{SU}(3)$, the free-field dimension of a baryon operator is $D_{B \text {,free }}=3(3 / 2)=9 / 2$, so, with Eq. (1.1), the lower bound (5.1) implies the upper bound on the anomalous dimension

$$
\mathrm{SU}(3): \gamma_{B}^{\left(j_{1}, j_{2}\right)} \leq \frac{7}{2}-\left(j_{1}+j_{2}\right)
$$

Specifically, for the various operators considered here (suppressing \pm ),

$$
\begin{aligned}
& \gamma_{B}^{\left(\frac{1}{2}, 0\right)} \leq 3 \\
& \gamma_{B}^{\left(\frac{3}{2}, 0\right)} \leq 2
\end{aligned}
$$

and 


$$
\gamma_{B}^{\left(1, \frac{1}{2}\right)} \leq 2
$$

For the present theory with gauge group $\mathrm{SU}(3)$ and $N_{f}$ fermions in the fundamental representation, the previous work in [34-37] led to the inference that the lower end of the NACP occurs at $N_{f, c r}$ around 8-9. In Fig. 1 and Fig. 2, one can see that our scheme-independent calculations of $\gamma_{B}^{\left(\frac{1}{2}, 0\right)+}$ and $\gamma_{B}^{\left(\frac{1}{2}, 0\right)-}$ to $O\left(\Delta_{f}^{3}\right)$ are well below the upper bound of 3 in (5.3). Our results for $\gamma_{B}^{\left(\frac{3}{2}, 0\right)+}$ and $\gamma_{B}^{\left(1, \frac{1}{2}\right)-}$ are negative, so they obviously also satisfy the respective upper bounds (5.4) and (5.5).

The fact that these baryon anomalous dimensions, as calculated to $O\left(\Delta_{f}^{3}\right)$, do not saturate their respective unitarity upper bounds as $N_{f}$ decreases toward the lower end of the non-Abelian Coulomb phase is reminiscent of the situation for an $\mathcal{N}=1$ supersymmetric gauge theory with gauge group $\mathrm{SU}\left(N_{c}\right)$ and $N_{f}$ pairs of chiral superfields, transforming respectively as the representations $R$ and $\bar{R}$ of $\mathrm{SU}\left(N_{c}\right)$, as studied in [39]. For this supersymmetric gauge theory, the only composite chiral superfield for which the anomalous dimension saturates its unitarity upper bound from conformal invariance as $N_{f}$ approaches the lower end of the NACP from above is the gauge-invariant quadratic chiral superfield, which contains the $\bar{\psi} \psi$ component field product. In contrast [aside from the pseudoreal case of SU(2)], a baryonic chiral superfield does not saturate its unitarity upper bound from conformal invariance at the lower end of the NACP [39].

\section{SCHEMES FOR ILLUSTRATIVE CALCULATIONS}

In this section we review some background and methods relevant for our calculations illustrating the scheme independence of the $\Delta_{f}$ series expansions for $\gamma_{\bar{\psi} \psi, \text { IR }}$ and $\beta_{\mathrm{IR}}^{\prime}$. We consider several schemes for regularization and renormalization. We first discuss these schemes. Recall that a common expression that one obtains from loop integrals performed in $d$-dimensional spacetime is

$$
\frac{\Gamma(2-(d / 2))}{(4 \pi)^{d / 2}} \frac{1}{A^{(d / 2)-2}},
$$

where $\Gamma(z)$ is the Euler gamma function, and $A$ is a denominator depending on some external momenta. Defining $\epsilon=4-d$ and expanding about $\epsilon=0$, using the Taylor-Laurent expansion of $\Gamma(z)$ about a pole at $z=0$,

$$
\Gamma(z)=\frac{1}{z}-\gamma_{E}+O(z)
$$

Eq. (6.1) becomes

$$
\frac{1}{(4 \pi)^{2}}\left[\frac{2}{\epsilon}-\gamma_{E}+\ln (4 \pi)-\ln A+O(\epsilon)\right]
$$

where

$$
\gamma_{E}=\lim _{n \rightarrow \infty}\left(\sum_{k=1}^{n} \frac{1}{k}-\ln n\right) \simeq 0.5772157
$$

In the minimal subtraction scheme MS [14], one subtracts the pole term, 2/ $\epsilon$. In the modified minimal subtraction scheme $\overline{\mathrm{MS}}$ [13], one subtracts the pole term and also the two following terms, namely the combination $2 / \epsilon-\gamma_{E}+\ln (4 \pi)$. Both the MS and $\overline{\mathrm{MS}}$ schemes are mass-independent and have the appeal that the beta function and anomalous dimensions of gauge-invariant operators are gauge-invariant. As was noted above, the calculations of $[33-38,40,41]$ used this scheme, although the resulting $\Delta_{f}$ expansions were proved to be schemeindependent.

In addition to the $\overline{\mathrm{MS}}$ scheme used in the previous work [34-38,40], the schemes that we use for our present illustrative demonstrations of scheme independence of $\Delta_{f}$ expansions are the following:

(1) the modified renormalization-invariant scheme $\left(\mathrm{RI}^{\prime}\right)$ $[63,64]$,

(2) the momentum subtraction scheme MOMggg defined by focusing on the triple-gluon vertex [29,70],

(3) the momentum subtraction scheme MOMh defined by focusing on the gluon-ghost-ghost vertex $[29,70]$,

(4) the momentum subtraction scheme MOMq defined by focusing on the gluon-fermion-fermion vertex $[29,70]$ (indicated with the subscript $q$ for "quark"), and

(5) the minimal momentum subtraction (mMOM) scheme $[65,68]$.

We write the conventional expansion of $\gamma_{\bar{\psi} \psi}$ as

$$
\gamma_{\bar{\psi} \psi}=\sum_{\ell=1}^{\infty} c_{\ell} a^{\ell},
$$

where the $c_{\ell}$ are the $\ell$-loop coefficients and, where no confusion will result, we set $c_{\ell} \equiv c_{\bar{\psi} \psi \mu, \ell}$. The one-loop coefficient, $c_{1}=6 C_{f}$, is scheme-independent, while the $c_{\ell}$ with $\ell \geq 2$ are scheme-dependent [44]. The evaluation of the $n$-loop truncation of (6.5) at the IRFP is obtained by substituting $\alpha=\alpha_{\mathrm{IR}, n \ell}$ and is denoted $\gamma_{\mathrm{IR}, n \ell}$.

Concerning the beta function (1.2), the one-loop coefficient, $b_{1}$ [3], is scheme-independent. In mass-independent schemes, the two-loop coefficient, $b_{2}$ [4], is also independent of the specific scheme [44]. We have mentioned above the calculations of $b_{3}$ [11], $b_{4}$ [12], and $b_{5}[16,17]$ in the $\overline{\mathrm{MS}}$ scheme. As noted, the $c_{\ell}$ were calculated to four-loop order [26] and to five-loop order in [27], in the $\overline{\mathrm{MS}}$ scheme [78]. 
The $b_{\ell}$ and $c_{\ell}$ have been calculated to four-loop order in the RI' scheme [64] and the minimial MOM (mMOM) scheme [68]. Additional calculations in generalized MOM schemes were presented in [70]. A comparison of conventional calculations of $\alpha_{\mathrm{IR}, n \ell}$ and $\gamma_{\mathrm{IR}, n \ell}$ was given up to the four-loop order in $[20,21,23,25]$. An important aspect in which the $\mathrm{RI}^{\prime}$ and MOM schemes differ with the $\overline{\mathrm{MS}}$ scheme is that beyond the lowest orders, the $b_{\ell}$ and $c_{\ell}$ are gauge-dependent. We consider a covariant gauge-fixing term so that the gauge part of the Lagrangian is [with our (+---) metric]

$$
\mathcal{L}_{\text {gauge }}=-\frac{1}{4} F_{\mu \nu}^{a} F^{\mu \nu, a}-\frac{1}{2 \xi}\left(\partial^{\mu} A_{\mu}^{a}\right)^{2}+\text { F.P. },
$$

where

$$
F_{\mu \nu}^{a}=\partial_{\mu} A_{\nu}^{a}-\partial_{\nu} A_{\mu}^{a}+g f^{a b c} A_{\mu}^{b} A_{\nu}^{c}
$$

is the field-strength tensor, with $a=1, . ., o(G)$ is the group index, $o(G)$ is the order of the gauge group, $f^{a b c}$ are the structure constants of the Lie algebra of $G$, and F.P. denote Faddeev-Popov terms. The gauge field propagator is thus

$$
\Delta_{\mu \nu}^{a b}(k)=-\frac{\delta^{a b}\left[g_{\mu \nu}-(1-\xi) \frac{k_{\mu} k_{\nu}}{k^{2}}\right]}{k^{2}} .
$$

The Landau gauge corresponds to $\xi=0$, where this propagator is transverse, i.e., $k^{\mu} \Delta_{\mu \nu}^{a b}(k)=0$. In these other schemes, the gauge parameter $\xi$ also depends on the Euclidean scale $\mu$, and so there is an associated function that measures this dependence, namely

$$
\beta_{\xi}=\frac{d \xi}{d \ln \mu} .
$$

We write the series expansion for this in powers of the coupling as

$$
\beta_{\xi}=-2 \xi \sum_{\ell=1}^{\infty} b_{\xi, \ell} a^{\ell}
$$

Evidently, the situation is the simplest in Landau gauge, since in this gauge, $\beta_{\xi}=0$ and the gauge parameter is independent of the Euclidean scale. The value of $\alpha$ at the IR zero of $\beta_{\alpha}$ and the resultant value of $\gamma_{\bar{\psi} \psi, \text { IR }}$ were calculated in Landau gauge at the three-loop level in the RI' scheme in [20] and in the mMOM scheme in [21], and at the four-loop level in [23]. We recall the procedure for this calculation. One looks for a physically acceptable simultaneous solution to the two coupled equations

$$
\beta_{\alpha}(\alpha, \xi)=0, \quad \beta_{\xi}(\alpha, \xi)=0,
$$

where we have explicitly indicated the dependence of $\beta_{\alpha}$ and $\beta_{\xi}$ on the variables $\alpha$ and $\xi$. Because $\beta_{\xi}$ is proportional to $\xi$, one is always guaranteed to find a solution with $\xi=0$. That is, if $\xi=0$ at some value $\mu=\mu_{0}$, then $\xi=0$ for all $\mu$. This was the basis for the choice of Landau gauge in Refs. [20,21,23]. As was discussed in [23], there also exist fixed points for which $\xi \neq 0$, but these solutions are on a different footing from the $\xi=0$ solution. As was noted in [20], at the two-loop level in the mMOM scheme, there is also an IRFP with $\xi_{2 \ell}=-3$, and calculations at the threeloop level exhibit an IRFP with $\xi_{3 \ell}$ near to this value (see also [79]). A list of the $b_{\ell}, b_{\xi, \ell}$, and $c_{\ell}$ for general $\xi$, with $1 \leq \ell \leq 3$ in the mMOM scheme was given in [20] and a list of the $b_{\ell}, b_{\xi, \ell}$, and $c_{\ell}$ for $\xi=0$, i.e., Landau gauge, with $1 \leq \ell \leq 4$ was given in [23] for the $\mathrm{RI}^{\prime}$ and $\mathrm{mMOM}$ schemes. We will also remark on the general case in which $\xi$ is not necessarily zero. The corresponding expressions for the $b_{\ell}, c_{\ell}$, and $b_{\xi, \ell}$ are too long and complicated to include here; they have been given, for example, as external files with the arXiv version of [70]. An important difference between the $c_{\ell}$ in the $\mathrm{RI}^{\prime}$ scheme and the $b_{\ell}$ and $c_{\ell}$ in the MOM schemes, as contrasted with the $b_{\ell}$ and $c_{\ell}$ in the $\overline{\mathrm{MS}}$ scheme is that in the non-MS schemes, these coefficients depend on a number of additional mathematical functions and constants. For example, as was discussed in [25], at the four-loop level, in addition to the dependence on the group invariants $C_{A}, C_{f}$, and $T_{f}$, the $b_{\ell}$ and $c_{\ell}$ in the $\overline{\mathrm{MS}}, \mathrm{RI}^{\prime}$, and mMOM schemes contain dependence on the quantities

$$
\left\{\mathbb{Q}, \zeta_{3}, \zeta_{5}\right\}
$$

For the following, note that $\zeta_{m}$ with even $m=2 r$ are proportional to $\pi^{2 r}$ :

$$
\zeta_{2 r}=\frac{(-1)^{r+1} B_{2 r}(2 \pi)^{2 r}}{2(2 r) !}
$$

where the $B_{n}$ are the Bernoulli numbers, defined by

$$
\frac{t}{e^{t}-1}=\sum_{n=0}^{\infty} B_{n} \frac{t^{n}}{n !}
$$

so listing $\pi^{2}$ is equivalent to listing $\zeta_{2}$, etc. In contrast to Eq. (6.12), even at the lower, three-loop level, $b_{\ell}$ and $c_{\ell}$ in the other MOM schemes have a considerably more complicated form, since they depend on the following set of mathematical functions and constants:

$$
\begin{aligned}
& \left\{\mathbb{Q}, \pi^{2}, \zeta_{3}, \pi^{4}, \psi^{\prime}(1 / 3), \psi^{\prime \prime \prime}(1 / 3), s_{2}(\pi / k), s_{3}(\pi / k),\right. \\
& \left.\frac{\pi \ln (3)}{\sqrt{3}}, \frac{\pi \ln (3)^{2}}{\sqrt{3}}, \frac{\pi^{3}}{\sqrt{3}}\right\},
\end{aligned}
$$

where here $k$ takes the values $k=2$ and $k=6 ; \psi(s)$ is the Euler $\psi$ function, $\psi(s)=d \ln [\Gamma(s)] / d s, \psi^{\prime}(s)=d \psi(s) / d s$; and $s_{n}(z)$ is defined as 


$$
s_{n}(z)=\frac{1}{\sqrt{3}} \operatorname{Im}\left[\operatorname{Li}_{n}\left(\frac{e^{i z}}{\sqrt{3}}\right)\right]
$$

where $\operatorname{Li}_{n}(z)$ is the polylogarithm function,

$$
\mathrm{Li}_{n}(z)=\int_{0}^{z} \frac{\operatorname{Li}_{n-1}(z)}{t} d t
$$

with $\operatorname{Li}_{0}(z)=z /(1-z)$ and $\operatorname{Li}_{1}(z)=-\ln (1-z)$. For $|z| \leq 1$, this function has the series representation

$$
\operatorname{Li}_{n}(z)=\sum_{j=1}^{\infty} \frac{z^{j}}{j^{n}}, n=2,3, \ldots
$$

As noted above, the calculation of the coefficient $d_{n}$ in Eq. (1.11) requires, as input, the $\ell$-loop coefficients $b_{\ell}$ with $1 \leq \ell \leq n$. The calculation of the coefficient $\kappa_{n}$ in Eq. (1.8) requires, as inputs, the values of the $b_{\ell}$ for $1 \leq \ell \leq n+1$, and the $\ell$-loop coefficients $c_{\ell}$ in Eq. (6.5) with $1 \leq \ell \leq n$.

In addition to our explicit demonstration that different schemes yield the same values for the coefficients $d_{n}$ and $\kappa_{n}$ in the scheme-independent expansions (1.11) and (1.8), our work shows that the full physical content of these schemeindependent coefficients is derived from the use of the simplest scheme, namely $\overline{\mathrm{MS}}$. Thus, there is a huge cancellation of the additional mathematical functions and quantities in (6.15) in the scheme-independent coefficients $d_{n}$ and $\kappa_{n}$. On the one hand, one may take the view that this had to be true, since a rigorous proof was given already that these coefficients are scheme-independent and their values were therefore already completely determined from the calculations in [33-37] in the $\overline{\mathrm{MS}}$ scheme. But nevertheless, our explicit demonstration of the cancellation is quite a striking result.

\section{SCHEME-INDEPENDENT EXPANSION OF $\gamma_{\bar{\psi} \psi, \mathrm{IR}}$}

The coefficients $\kappa_{n}$ in the scheme-independent expansion of $\gamma_{\bar{\psi} \psi, \text { IR }}$ in powers of $\Delta_{f}$, Eq. (1.8), were calculated for a gauge group $G$ with $N_{f}$ fermions in a representation $R$ up to $n=3$ in [33] and up to $n=4$ in [36,37]. [The coefficient $\kappa_{4}$ was calculated for $G=\mathrm{SU}(3)$ and $R=F$ in [34].] For example, the first two of these coefficients are

$$
\kappa_{1}=\frac{8 C_{f} T_{f}}{C_{A}\left(7 C_{A}+11 C_{f}\right)}
$$

and

$$
\kappa_{2}=\frac{4 C_{f} T_{f}^{2}\left(5 C_{A}+88 C_{f}\right)\left(7 C_{A}+4 C_{f}\right)}{3 C_{A}^{2}\left(7 C_{A}+11 C_{f}\right)^{3}} .
$$

For the present work we have explicitly verified that we obtain the same results for these $\kappa_{n}$ using the RI', mMOM, and other MOM schemes. We have carried out this check to the highest order possible with existing inputs available in these schemes, i.e., to order $n=3$.

\section{SCHEME-INDEPENDENT EXPANSION OF $\beta_{\text {IR }}^{\prime}$}

The derivative $\beta_{\mathrm{IR}}^{\prime}$ is an important physical quantity characterizing the conformal field theory at $\alpha_{\mathrm{IR}}$. For general gauge group $G$ with $N_{f}$ fermions in a general representation $R$, the scheme-independent coefficients $d_{n}$ were calculated up to $n=4$ in [35] and up to $n=5$ in $[36,37]$. The first two nonzero coefficients are

$$
d_{2}=\frac{2^{5} T_{f}^{2}}{3^{2} C_{A}\left(7 C_{A}+11 C_{f}\right)}
$$

and

$$
d_{3}=\frac{2^{7} T_{f}^{3}\left(5 C_{A}+3 C_{f}\right)}{3^{3} C_{A}^{2}\left(7 C_{A}+11 C_{f}\right)^{2}} .
$$

We have explicitly verified that we obtain the same results for $d_{n}$ with the $\mathrm{RI}^{\prime}, \mathrm{mMOM}$, and other MOM schemes. We have carried out this check to the highest order possible with existing inputs available in these schemes, i.e., to order $n=4$.

\section{CONCLUSIONS}

In conclusion, in this paper we have presented the first analytic scheme-independent expansions to $O\left(\Delta_{f}^{3}\right)$ for the anomalous dimensions of a variety of (gauge-invariant) baryon operators at an infrared fixed point of an asymptotically free $\mathrm{SU}(3)$ gauge theory with $N_{f}$ fermions in the fundamental (triplet) representation. Furthermore, for an asymptotically free theory with a general gauge group $G$ and $N_{f}$ fermions in a general representation $R$ of $G$, we have given explicit illustrative demonstrations of the scheme independence of $\gamma_{\bar{\psi} \psi, \mathrm{IR}}$ and $\beta_{\mathrm{IR}}^{\prime}$ at an IRFP. Although this scheme independence had been proved rigorously earlier, it is worthwhile to see how different schemes yield identical results for the coefficients in the scheme-independent expansions. We have carried out these calculations for the $\mathrm{RI}^{\prime}$ and several MOM schemes.

\section{ACKNOWLEDGMENTS}

We thank R.M. Simms and L. Vecchi for useful discussions. This research was supported in part by U.K. Science and Technology Facilities Council (STFC) through the Consolidated Grant ST/L000431/1 and a Deutsche Forschungsgemeinschaft (DFG) Mercator Fellowship (J. A. G.), the Danish National Research Foundation grant DNRF90 to CP3-Origins at SDU (T. A. R.) and the U.S. National Science Foundation Grant NSF-PHY-16-1620628 (R. S.). This research was initiated at the Simons Workshop [48] in January 2018, and the authors are grateful to the Simons Foundation for funding this workshop. 
[1] Some early analyses of connections between scale and conformal invariance includeA. Salam, Ann. Phys. (N.Y.) 53, 174 (1969); A. M. Polyakov, JETP Lett. 12, 381 (1970); D. J. Gross and J. Wess, Phys. Rev. D 2, 753 (1970); C. G. Callan, S. Coleman, and R. Jackiw, Ann. Phys. (N.Y.) 59, 42 (1970). More recent works include J. Polchinski, Nucl. Phys. B303, 226 (1988); J.-F. Fortin, B. Grinstein, and A. Stergiou, J. High Energy Phys. 01 (2013) 184; A. Dymarsky, Z. Komargodski, A. Schwimmer, and S. Thiessen, J. High Energy Phys. 10 (2015) 171 and references therein.

[2] Our assumption that the fermions are massless does not entail any loss of generality, since if a fermion had a nonzero mass $m_{0}$, it would be integrated out of the low-energy effective field theory at scales $\mu<m_{0}$, and hence would not affect the properties at the infrared fixed point.

[3] D. J. Gross and F. Wilczek, Phys. Rev. Lett. 30, 1343 (1973); H. D. Politzer, Phys. Rev. Lett. 30, 1346 (1973).

[4] W. E. Caswell, Phys. Rev. Lett. 33, 244 (1974); D. R. T. Jones, Nucl. Phys. B75, 531 (1974).

[5] For the representation $R$ of the Lie group $G$, the quadratic Casimir invariant $C_{2}(R)$ is given by $T_{R}^{a} T_{R}^{a}=C_{2}(R) I$, where $I$ is the $\operatorname{dim}(R) \times \operatorname{dim}(R)$ identity matrix. For $R$ equal to the adjoint representation, we denote $C_{2}(a d j) \equiv C_{2}(G) \equiv C_{A}$. The trace invariant is $\operatorname{Tr}_{R}\left(T_{R}^{a} T_{R}^{b}\right)=T(R) \delta^{a b}$. For a fermion $f$ transforming according to a representation $R$, we often use the equivalent compact notation $T_{f} \equiv T(R)$ and $C_{f} \equiv C_{2}(R)$. We use standard normalizations, so that for $\operatorname{SU}\left(N_{c}\right), C_{A}=N_{c}$ and for $R$ equal to the fundamental representation, $C_{f}=\left(N_{c}^{2}-1\right) /\left(2 N_{c}\right)$ and $T_{f}=1 / 2$.

[6] We often implicitly consider $N_{f}$ to be generalized from the non-negative integers to the non-negative real numbers. If an expression for $N_{f}$ formally evaluates to a nonintegral real value, it is understood implicitly that one infers an appropriate integral value from it.

[7] T. Banks and A. Zaks, Nucl. Phys. B196, 189 (1982).

[8] T. A. Ryttov and R. Shrock, Phys. Rev. D 83, 056011 (2011).

[9] C. Pica and F. Sannino, Phys. Rev. D 83, 035013 (2011).

[10] R. Shrock, Phys. Rev. D 87, 105005 (2013); 87, 116007 (2013).

[11] O. V. Tarasov, A. A. Vladimirov, and A. Yu. Zharkov, Phys. Lett. B 93, 429 (1980); S. A. Larin and J. A. M. Vermaseren, Phys. Lett. B 303, 334 (1993).

[12] T. van Ritbergen, J. A. M. Vermaseren, and S. A. Larin, Phys. Lett. B 400, 379 (1997).

[13] W. A. Bardeen, A. J. Buras, D. W. Duke, and T. Muta, Phys. Rev. D 18, 3998 (1978).

[14] G. 't Hooft, Nucl. Phys. B61, 455 (1973).

[15] T. A. Ryttov and R. Shrock, Phys. Rev. D 94, 105015 (2016).

[16] P. A. Baikov, K. G. Chetyrkin, and J. H. Kühn, Phys. Rev. Lett. 118, 082002 (2017).

[17] F. Herzog, B. Ruijl, T. Ueda, J. A. M. Vermaseren, and A. Vogt, J. High Energy Phys. 02 (2017) 090.

[18] T. A. Ryttov and R. Shrock, Phys. Rev. D 86, 065032 (2012); 86, 085005 (2012).

[19] R. Shrock, Phys. Rev. D 88, 036003 (2013).

[20] T. A. Ryttov, Phys. Rev. D 89, 016013 (2014).

[21] T. A. Ryttov, Phys. Rev. D 89, 056001 (2014).

[22] R. Shrock, Phys. Rev. D 90, 045011 (2014).
[23] T. A. Ryttov, Phys. Rev. D 90, 056007 (2014).

[24] G. Choi and R. Shrock, Phys. Rev. D 90, 125029 (2014); 94, 065038 (2016).

[25] J. A. Gracey and R. M. Simms, Phys. Rev. D 91, 085037 (2015).

[26] K. G. Chetyrkin, Phys. Lett. B 404, 161 (1997); J. A. M. Vermaseren, S. A. Larin, and T. van Ritbergen, Phys. Lett. B 405, 327 (1997).

[27] P. A. Baikov, K. G. Chetyrkin, and J. H. Kühn, J. High Energy Phys. 10 (2014) 076; 04 (2017) 119.

[28] Recent reviews of higher-order QCD calculations include J. M. Campbell et al., arXiv:1310.5189; N. Brambilla et al., Eur. Phys. J. C 74, 2981 (2014); G. Heinrich, arXiv: 1710.04998.

[29] W. Celmaster and R. J. Gonsalves, Phys. Rev. Lett. 42, 1435 (1979); Phys. Rev. D 20, 1420 (1979).

[30] P. M. Stevenson, Phys. Lett. 100B, 61 (1981); Phys. Rev. D 23, 2916 (1981); Nucl. Phys. B868, 38 (2013) and references therein.

[31] S. J. Brodsky, G. P. Lepage, and P. B. Mackenzie, Phys. Rev. D 28, 228 (1983); S. J. Brodsky and X. G. Wu, Phys. Rev. Lett. 109, 042002 (2012); Phys. Rev. D 85, 034038 (2012); S. J. Brodsky, M. Mojaza, and X.-G. Wu, Phys. Rev. D 89, 014027 (2014) and references therein.

[32] G. Grunberg, Phys. Rev. D 46, 2228 (1992); E. Gardi and M. Karliner, Nucl. Phys. B529, 383 (1998); E. Gardi and G. Grunberg, J. High Energy Phys. 03 (1999) 024.

[33] T. A. Ryttov, Phys. Rev. Lett. 117, 071601 (2016).

[34] T. A. Ryttov and R. Shrock, Phys. Rev. D 94, 105014 (2016).

[35] T. A. Ryttov and R. Shrock, Phys. Rev. D 94, 125005 (2016).

[36] T. A. Ryttov and R. Shrock, Phys. Rev. D 95, 085012 (2017).

[37] T. A. Ryttov and R. Shrock, Phys. Rev. D 95, 105004 (2017).

[38] T. A. Ryttov and R. Shrock, Phys. Rev. D 96, 105015 (2017).

[39] T. A. Ryttov and R. Shrock, Phys. Rev. D 96, 105018 (2017).

[40] T. A. Ryttov and R. Shrock, Phys. Rev. D 97, 025004 (2018).

[41] T. A. Ryttov and R. Shrock, Phys. Rev. D 97, 016020 (2018).

[42] T. A. Ryttov and R. Shrock, Phys. Rev. D 97, 065020 (2018).

[43] J. A. Gracey, Phys. Lett. B 488, 175 (2000).

[44] D. J. Gross, in Methods in Field Theory, Les Houches 1975, edited by R. Balian and J. Zinn-Justin (North Holland, Amsterdam, 1976), p. 141.

[45] S. L. Adler, J. C. Collins, and A. Duncan, Phys. Rev. D 15, 1712 (1977); J. C. Collins, A. Duncan, and S. Joglekar, Phys. Rev. D 16, 438 (1977); N. K. Nielsen, Nucl. Phys. B120, 212 (1977); see also H. Kluberg-Stern and J.-B. Zuber, Phys. Rev. D 12, 467 (1975).

[46] S. S. Gubser, A. Nellore, S. S. Pufu, and E. D. Rocha, Phys. Rev. Lett. 101, 131601 (2008).

[47] Recent reviews include the Lattice for BSM 2017 Workshop at http://www-hep.colorado.edu/ eneil/lbsm17; Lattice2017 at http://wpd.ugr.es/ lattice2017, and [48]. 
[48] Simons Workshop on Continuum and Lattice Approaches to the Infrared Behavior of Conformal and Quasiconformal Gauge Theories, Jan. 8-12, 2018, T. A. Ryttov and R. Shrock, organizers; http://scgp.stonybrook.edu/archives/21358.

[49] G. Mack, Commun. Math. Phys. 55, 1 (1977); B. Grinstein, K. Intriligator, and I. Rothstein, Phys. Lett. B 662, 367 (2008); Y. Nakayama, Phys. Rep. 569, 1 (2015).

[50] B. Holdom, Phys. Lett. 150B, 301 (1985); K Yamawaki, M. Bando, and K. Matumoto, Phys. Rev. Lett. 56, 1335 (1986); T. Appelquist, D. Karabali, and L. C. R. Wijewardhana, Phys. Rev. Lett. 57, 957 (1986).

[51] W. Goldberger, B. Grinstein, and W. Skiba, Phys. Rev. Lett. 100, 111802 (2008).

[52] Lattice studies of quasiconformal theories have shown the appearance of a light scalar; see, e.g., Y. Aoki et al. (LatKMI Collaboration), Phys. Rev. D 89, 111502 (2014), 96, 014508 (2017); T. Appelquist et al. (LSD Collaboration), Phys. Rev. D 93, 114514 (2016); Z. Fodor et al. (Lattice Higgs Collaboration), Proc. Sci., LATTICE2015, 219 (2016) [arXiv:1605.08750].

[53] For some recent studies of these types of ultraviolet completions, see, e.g., T. A. Ryttov and R. Shrock, Phys. Rev. D 81, 115013 (2010); Eur. Phys. J. C 71, 1523 (2011); T. Appelquist and R. Shrock, Phys. Rev. D 88, 105012 (2013); Y. Shi and R. Shrock, Phys. Rev. D 91, 045004 (2015); 92, 125009 (2015); 92, 105032 (2015), and references therein.

[54] M. Peskin, Phys. Lett. 88B, 128 (1979).

[55] A. A. Pivovarov and L. R. Surguladze, Nucl. Phys. B360, 97 (1991).

[56] V. M. Braun, S. E. Derkachov, and A. N. Manashov, Phys. Rev. Lett. 81, 2020 (1998).

[57] S. Kränkl and A. Manashov, Phys. Lett. B 703, 519 (2011).

[58] J. A. Gracey, J. High Energy Phys. 09 (2012) 052.

[59] With the definition (1.1), our Eq. (3.1) involves a multiplication by a factor of 2 relative to Eq. (6.13) in [58].

[60] C. Pica and F. Sannino, Phys. Rev. D 94, 071702(R) (2016).

[61] L. Vecchi, arXiv:1607.02740.

[62] In contrast, in the QCD-like phase with confinement, even if the two-loop beta function has an IR zero, this is not exact. The reason is that there is spontaneous chiral symmetry breaking $(\mathrm{S} \chi \mathrm{SB})$ in this $\mathrm{QCD}$-like phase, which produces dynamical masses for the fermions. Consequently, the fermions are integrated out of the low-energy effective theory as one evolves further toward the infrared, and the beta function becomes that of a pure gauge theory, which has no IR zero.

[63] G. Martinelli, C. Pittori, C. T. Sachrajda, M. Testa, and A. Vladikas, Nucl. Phys. B445, 81 (1995).

[64] J. A. Gracey, Nucl. Phys. B662, 247 (2003).

[65] L. von Smekal, K. Maltman, and A. Sternbeck, Phys. Lett. B 681, 336 (2009).

[66] J. A. Gracey, Phys. Rev. D 84, 085011 (2011).

[67] J. A. Gracey, Phys. Lett. B 700, 79 (2011).

[68] J. A. Gracey, J. Phys. A 46, 225403 (2013).

[69] J. M. Bell and J. A. Gracey, Phys. Rev. D 88, 085027 (2013).

[70] J. A. Gracey and R. M. Simms, Phys. Rev. D 97, 085016 (2018).

[71] K. G. Chetyrkin, G. Falcioni, F. Herzog, and J. A. M. Vermaseren, J. High Energy Phys. 10 (2017) 179; 12 (2017) 006.

[72] W. Siegel, Phys. Lett. B 84, 193 (1979); 94, 37 (1980); D. M. Capper, D. R. T. Jones, and P. van Nieuwenhuizen, Nucl. Phys. B167, 479 (1980); W. Stöckinger, J. High Energy Phys. 03 (2005) 076.

[73] V. A. Novikov, M. A. Shifman, A. I. Vainshtein, and V. I. Zakharov (NSVZ), Phys. Lett. B 166, 329 (1986).

[74] N. Seiberg, Nucl. Phys. B435, 129 (1995).

[75] T. A. Ryttov and R. Shrock, Phys. Rev. D 85, 076009 (2012).

[76] R. Shrock, Phys. Rev. D 91, 125039 (2015); G. Choi and R. Shrock, Phys. Rev. D 93, 065013 (2016).

[77] A. L. Kataev and K. V. Stepanyantz, Phys. Lett. B 730, 184 (2014); Theor. Math. Phys. 181, 1531 (2014).

[78] Parenthetically, we note that although the conventional calculation of the $\bar{\psi} \psi$ anomalous dimension to five-loop order in [15] used $c_{5}$ from [27], the calculation of the scheme-independent coefficients $\kappa_{n}$ [33,34,36-38,40], which have been carried out up to $n=4$ order for general $G$ and $R$, did not make use of $c_{5}$. The reason is that, as mentioned in the text, the calculation of $\kappa_{n}$ requires, as input, the $b_{\ell}$ with $1 \leq \ell \leq n+1$ and the $c_{\ell}$ with $1 \leq \ell \leq n$. Hence, in order to use $c_{5}$ to calculate $\kappa_{5}$, one would also need $b_{6}$, but the $b_{\ell}$ have only been calculated up to $\ell=5$ loop order $[16,17]$.

[79] T. Appelquist, K. Lane, and U. Mahanta, Phys. Rev. Lett. 61, 1553 (1988). 